\title{
AVALIAÇÃo de PARÂMETROS DO MÉTODO DE ELEMENTOS DISCRETOS NA SIMULAÇÃO DE UM TAMBOR ROTATIVO DE BANCADA COM FERTILIZANTES
}

\author{
B.C.SILVERIO ${ }^{1 *}$, D.B.L.SANTOS ${ }^{2}$, I.A.RESENDE ${ }^{2}$, C. R. DUARTE ${ }^{2}$, K. G. SANTOS $^{3}$ e \\ M.A.S.BARROZO ${ }^{2}$ \\ ${ }^{1}$ Universidade Federal Goiás, Instituto de Química \\ ${ }^{2}$ Universidade Federal de Uberlândia, Faculdade de Engenharia Química \\ ${ }^{3}$ Universidade Federal do Triângulo Mineiro, Instituto de Ciências Tecnológicas e Exatas \\ E-mail para contato: beatrizcristinadejesus@hotmail.com
}

\begin{abstract}
RESUMO - O Método de Elementos Discretos (DEM) tem se mostrado uma técnica computacional cada vez mais utilizada para simular o comportamento de sistemas em fase discreta em sistemas particulados. Existem vários estudos de DEM para diferentes tipos de secadores e tambores rotativos, porém esses estudos são aplicados para diferentes tipos de materiais. Neste trabalho avaliou-se simulações do escoamento de fertilizantes em um tambor rotativo de bancada. As simulações foram feitas com diferentes combinações de valores de parâmetros para um modelo linear. Através da comparação dessas simulações com dados experimentais de ângulo dinâmico de repouso, foi possível avaliar o efeito da combinação de parâmetros para o escoamento de fertilizantes granulados.
\end{abstract}

\section{INTRODUÇÃO}

Um melhor entendimento dos fenômenos envolvidos na fluidodinâmica de um secador rotativo, necessariamente passa pelo desenvolvimento de uma modelagem com base nas equações de conservação de massa, momento e energia, com suas equações constitutivas pertinentes, bem como pelo acoplamento de pressão e velocidade.

A validação dos modelos empregados pode atenuar problemas de contato sólido-gás, ou mesmo indicar a necessidade de modificação da configuração e de condições operacionais, a fim de propiciar um melhor escoamento das partículas sólidas e um melhor contato gás-partícula.

A resolução numérica dos escoamentos multifásicos pode ser compreendida tanto pela abordagem Euler-Lagrange, quanto pela abordagem Euler-Euler. A abordagem Euler-Lagrange consiste em modelar a fase fluida como contínua utilizando as equações de Navier-Stokes calculadas no tempo, ausente da fase discreta, sendo assim, utiliza-se das informações fluidodinâmicas como dados de entrada para então fazer a descrição do comportamento da fase discreta.

A fase discreta é resolvida injetando-se um grande número de partículas, através do campo de 


\section{9 a 22 de outubro de 2014 \\ Florianópolis/SC}

escoamento calculado e são tratadas pela mecânica clássica do corpo sólido, especificamente pela aplicação da $2^{\mathrm{a}}$ Lei do movimento de Newton, sendo considerada a interação de momento, massa e energia da fase discreta com a fase fluida (Santos, 2007).

Dentre as dificuldades encontradas na abordagem Lagrangeana, podemos ressaltar a necessidade de utilizar correlações para descrever as interações partícula-parede, partícula-partícula e partícula-gás e a dificuldade em prever as variáveis de campo para a fase particulada, dificultando a visualização científica dos fenômenos que influenciam nas trajetórias das partículas.

Um método aplicado para a abordagem Lagrangeana consiste na utilização de modelos DEM (Discret Element Method) ou Método dos Elementos Discretos, que permite simular partículas em movimento como pontos de massa em sistemas concentrados.

Di Renzo (2004) comparou três modelos para forças de contato, e numa comparação macroscópica o modelo linear "mola-amortecedor" (spring-dashpot) estudado mostrou bons resultados para a predição das forças de contato.

A literatura mostra vários trabalhos de estudos e verificações de modelos que se utilizam de vários parâmetros, dependendo do processo e material analisados (Anand et al. 2009, Ketterhageng et al 2008, Liu et al. 2013, Zong et al, 2006). Alguns dos parâmetros utilizados, por exemplo, no modelo linear "mola-amortecedor", citado anteriormente, são os coeficientes de restituição $(\eta)$, de fricção $(\mu)$ e o coeficiente de elasticidade ou mola $(\mathrm{k})$.

Nota-se que os valores encontrados para os parâmetros dos modelos nos trabalhos de simulação DEM, variam significativamente, pois cada estudo foi concebido para equipamentos distintos e representa o escoamento de materiais diferentes os quais podem conduzir diferentes respostas de colisões partícula-partícula e partícula-parede.

Desta forma, o objetivo deste trabalho foi realizar simulações utilizando o método DEM e avaliar diferentes valores dos parâmetros: coeficientes de restituição $(\eta)$, de fricção $(\mu)$ e o coeficiente de elasticidade ou mola $(\mathrm{k})$ do modelo linear "mola-amortecedor" para encontrar um melhor conjunto de parâmetros que melhor caracterize o sistema em estudo.

\section{METODOLOGIA}

\subsection{Procedimento Experimental}

. O experimento foi realizado em um cilindro rotativo de acrílico, com $0,1 \mathrm{~m}$ de diâmetro e 0,1 $\mathrm{m}$ de altura. A massa interna de fertilizantes introduzida foi $415 \mathrm{~g}$ de forma que $50 \%$ do volume do tambor fosse preenchido, e a velocidade rotacional do sistema era de $25 \mathrm{rpm}$.

O ângulo dinâmico de repouso foi obtido com resultados de 15 fotografias do experimento utilizando-se o software GLOBAL Lab® IMAGE/2. 


\section{9 a 22 de outubro de 2014 \\ Florianópolis/SC}

\subsection{Metodologia de simulação}

O software FLUENT® 14.0 foi utilizado nas simulações para a obtenção do ângulo dinâmico de repouso tambor rotativo de bancada na avaliação dos parâmetros do Método dos Elementos Discretos (DEM) secadores rotativos.

A malha computacional foi obtida a partir do software GAMBIT® 2.3.16 e foi construída de forma a se garantir que o tamanho das células fosse no mínimo três vezes maior do que o diâmetro da esfera de igual volume da partícula (dp).

Uma vez que se opta pela utilização do modelo Euleriano, o software FLUENT ANSYS® 14.0, além de resolver as equações de transporte para a fase contínua, permite simular uma fase discreta segundo uma abordagem Lagrangeana que utiliza o DEM (Discret Element Method). Esta segunda fase consiste em partículas esféricas. $\mathrm{O}$ acoplamento entre as fases e o seu impacto sobre ambas as trajetórias da fase discreta e o fluxo de fase contínua também é incluído nos cálculos.O modelo utilizado resolve para a fase fluida a equação da Continuidade e a equação do Movimento.

As simulações DEM foram feitas utilizando-se malhas construídas em três dimensões. O conjunto de equações de conservação e constitutivas foi resolvido utilizando-se o método dos volumes finitos. $\mathrm{O}$ acoplamento entre velocidade e pressão foi definido pelo algoritmo SIMPLE. Os resíduos utilizados foram menores que $10^{-3}$. Na parede foi considerada uma condição de contorno de não deslizamento. Para a discretização espacial foi utilizado o modelo QUICK e para a discretização temporal o modelo implícito de primeira ordem.

Nas simulações do secador rotatório com suspensores foi necessário utilizar uma condição de malha móvel, em que toda a malha se movimenta à uma mesma velocidade rotacional. $\mathrm{O}$ modelo mais geral para o fluxo em zonas móveis com deformação celular no FLUENT ANSYS® é o modelo de malha móvel.

A fim de avaliar o efeito dos parâmetros do modelo DEM sobre o ângulo dinâmico de repouso nos suspensores, bem como na carga de fertilizantes nos suspensores, foram realizadas simulações previstas por meio de um planejamento composto central ortogonal com 7 réplicas no ponto central, conforme mostra a Tabela 1(simulação 15 são os valores do ponto central).

Os valores dos parâmetros escolhidos tiveram como base a ampla faixa encontrada na revisão da literatura. Sendo assim, nem todas as combinações entre os parâmetros necessariamente conduzem a resultados fisicamente coerentes, mas pode contribuir para verificar o efeito dos parâmetros e suas interações.

A Tabela 2 apresenta as condições utilizadas nas simulações do ângulo dinâmico de repouso do tambor rotativo de bancada. 
Tabela 1 - Planejamento composto central para avaliação dos parâmetros.

\begin{tabular}{|c|c|c|c|c|c|c|}
\hline \multirow[b]{2}{*}{ Simulação } & \multicolumn{3}{|c|}{$\begin{array}{c}\text { Variáveis } \\
\text { Codificadas }\end{array}$} & \multicolumn{3}{|c|}{$\begin{array}{l}\text { Valores dos } \\
\text { Parâmetros }\end{array}$} \\
\hline & $\mathrm{v}_{1}(k)$ & $\mathrm{v}_{2}\left(\mu_{f}\right)$ & $\mathrm{v}_{3}(\eta)$ & $k[\mathrm{~N} / \mathrm{m}]$ & $\mu_{f}$ & $\eta$ \\
\hline 1 & $-1,00$ & $-1,00$ & $-1,00$ & 400 & 0,2000 & 0,2000 \\
\hline 2 & $-1,00$ & $-1,00$ & 1,00 & 400 & 0,2000 & 0,8000 \\
\hline 3 & $-1,00$ & 1,00 & $-1,00$ & 400 & 0,8000 & 0,2000 \\
\hline 4 & $-1,00$ & 1,00 & 1,00 & 400 & 0,8000 & 0,8000 \\
\hline 5 & 1,00 & $-1,00$ & $-1,00$ & 1600 & 0,2000 & 0,2000 \\
\hline 6 & 1,00 & $-1,00$ & 1,00 & 1600 & 0,2000 & 0,8000 \\
\hline 7 & 1,00 & 1,00 & $-1,00$ & 1600 & 0,8000 & 0,2000 \\
\hline 8 & 1,00 & 1,00 & 1,00 & 1600 & 0,8000 & 0,8000 \\
\hline 9 & $-1,58$ & 0,00 & 0,00 & 54,98 & 0,5000 & 0,5000 \\
\hline 10 & 1,58 & 0,00 & 0,00 & 1945 & 0,5000 & 0,5000 \\
\hline 11 & 0,00 & $-1,58$ & 0,00 & 1000 & 0,0275 & 0,5000 \\
\hline 12 & 0,00 & 1,58 & 0,00 & 1000 & 0,9725 & 0,5000 \\
\hline 13 & 0,00 & 0,00 & $-1,58$ & 1000 & 0,5000 & 0,0275 \\
\hline 14 & 0,00 & 0,00 & 1,58 & 1000 & 0,5000 & 0,9725 \\
\hline 15 & 0,00 & 0,00 & 0,00 & 1000 & 0,5000 & 0,5000 \\
\hline 16 & 0,00 & 0,00 & 0,00 & 1000 & 0,5000 & 0,5000 \\
\hline 17 & 0,00 & 0,00 & 0,00 & 1000 & 0,5000 & 0,5000 \\
\hline 18 & 0,00 & 0,00 & 0,00 & 1000 & 0,5000 & 0,5000 \\
\hline 19 & 0,00 & 0,00 & 0,00 & 1000 & 0,5000 & 0,5000 \\
\hline 20 & 0,00 & 0,00 & 0,00 & 1000 & 0,5000 & 0,5000 \\
\hline 21 & 0,00 & 0,00 & 0,00 & 1000 & 0,5000 & 0,5000 \\
\hline
\end{tabular}

Tabela 2 - Condições adotadas nas simulações do secador rotatório convencional.

\begin{tabular}{|c|c|}
\hline time step fase contínua $(\mathrm{s})$ & $\mathbf{0 , 0 0 1}$ \\
time step fase discreta $(\mathrm{s})$ & $\mathbf{0 , 0 0 0 1}$ \\
Velocidade rotacional $(\mathrm{rpm})$ & $\mathbf{2 5}$ \\
$\mathrm{N}^{\mathbf{o}}$ de partículas & $\mathbf{8 6 7 0}$ \\
$\mathrm{N}^{\mathrm{o}}$ de células da malha & $\mathbf{9 1 8}$ \\
Tempo simulado $(\mathrm{s})$ & $\mathbf{1 2}$ \\
$\mathrm{N}^{\mathrm{o}}$ de suspensores & $\mathbf{0}$ \\
Diâmetro do cilindro $(\mathrm{m})$ & $\mathbf{0 , 1}$ \\
Comprimento do cilindro $(\mathrm{m})$ & $\mathbf{0 , 1}$ \\
\hline
\end{tabular}


Os valores simulados dos ângulos dinâmicos de repouso foram então comparados com os valores obtidos experimentalmente nas mesmas condições.

Além de comparar os resultados do ângulo dinâmico de repouso obtido nas simulações aos dados experimentais, a escolha dos parâmetros de simulação que melhor caracterizam o escoamento do fertilizante em tambores rotativos baseou-se também na comparação visual qualitativa entre as imagens obtidas durante o experimento e os perfis simulados de posição das partículas.

\section{RESULTADOS E DISCUSSÕES}

A Figura 1 mostra a fotografia do tambor rotativo de bancada utilizado. $\mathrm{O}$ ângulo de repouso foi obtido para 15 fotografias do experimento. Observou-se que o ângulo de repouso médio do material obtido através de fotografias foi de $38,6^{\circ}$ com desvio de $3,5 \%$.

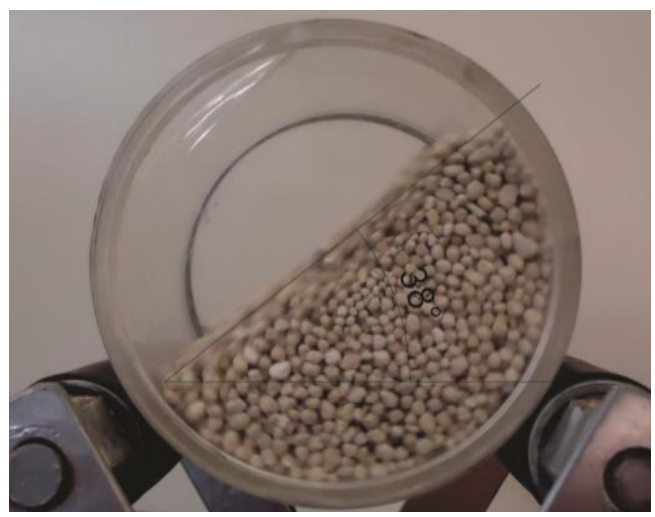

Figura 1 - Unidade experimental com tambor rotativo de acrílico.

Para avaliação dos parâmetros relacionados ao coeficiente de fricção, constante elástica, e coeficiente de restituição, nas simulações DEM do tambor rotativo de acrílico utilizou-se o PCC da Tabela 1, reproduzido na Tabela 3, com os resultados simulados do ângulo dinâmico de repouso $\phi$.

A Tabela 3 mostra os resultados de ângulo dinâmico de repouso para as simulações realizadas com diferentes valores de parâmetros conforme o PCC apresentado.

Esses resultados mostram uma visível sensibilidade do ângulo dinâmico de repouso para as diferentes combinações de parâmetros propostas. Enquanto algumas simulações apresentaram ângulos de repouso muito grandes, como a simulação 3 com $66,8^{\circ}$, outra simulação apresentou ângulo de repouso muito pequeno, simulação $11 \mathrm{com} 5,8^{\circ}$ sendo estas as simulações que apresentaram maiores erros relacionados ao valor experimental.

Os valores mais próximos ao encontrado experimentalmente, ou seja $38^{\circ}$, foram os valores obtidos nas simulações $1,2,5$ e 7 , com erro de até $3,7 \%$, sendo que o valor obtido pela simulação 5 foi o mais próximo do resultado encontrado experimentalmente. As demais simulações apresentaram resultados com erros experimentais muito grandes maiores do que $45 \%$. 
Esses resultados evidenciam a influência dos resultados das simulações a partir da combinação dos parâmetros usados. Observa-se nas simulações 1 e 2 que os resultados não obtiveram influência com o aumento do coeficiente $\eta$ de 0,2 para 0,8 , quando utilizados valores constantes de $k=400 \mathrm{~N} / \mathrm{m}$ e $\mu_{f}=0,2$. Já observando as simulações 5 e 7 observamos que o resultado da simulação foi pouco influenciado pela alteração do coeficiente $\mu_{f}$ de 0,2 para 0,8 , mantendo-se a combinação dos outrso dois parâmetros $k=1600 \mathrm{~N} / \mathrm{m}$ e $\eta=0,2$.

A Figura 2 mostra os resultados das simulações do método DEM utilizando-se o modelo "molaamortecedor", nas diferentes combinações de parâmetros do PCC. Nela, no canto superior está o número da simulação correspondente ao planejamento e no canto inferior direito o ângulo de repouso obtido.

Tabela 3 - Resultados do ângulo dinâmico de repouso para as simulações do PCC.

\begin{tabular}{|c|c|c|c|c|c|c|c|c|}
\hline \multirow[b]{2}{*}{ Simulação } & \multicolumn{3}{|c|}{$\begin{array}{c}\text { Variáveis } \\
\text { Codificadas }\end{array}$} & \multicolumn{3}{|c|}{$\begin{array}{l}\text { Valores dos } \\
\text { Parâmetros }\end{array}$} & \multirow[b]{2}{*}{$\phi\left({ }^{\circ}\right)$} & \multirow[b]{2}{*}{ erro(\%) } \\
\hline & $\mathrm{v}_{1}(k)$ & $\mathrm{v}_{2}\left(\mu_{f}\right)$ & $\mathrm{v}_{3}(\eta)$ & $k[\mathrm{~N} / \mathrm{m}]$ & $\mu_{f}$ & $\eta$ & & \\
\hline 1 & $-1,00$ & $-1,00$ & $-1,00$ & 400 & 0,2000 & 0,2000 & 37,2 & 3,8 \\
\hline 2 & $-1,00$ & $-1,00$ & 1,00 & 400 & 0,2000 & 0,8000 & 37,2 & 3,8 \\
\hline 3 & $-1,00$ & 1,00 & $-1,00$ & 400 & 0,8000 & 0,2000 & 66,8 & 72,9 \\
\hline 4 & $-1,00$ & 1,00 & 1,00 & 400 & 0,8000 & 0,8000 & 65,4 & 69,3 \\
\hline 5 & 1,00 & $-1,00$ & $-1,00$ & 1600 & 0,2000 & 0,2000 & 39,2 & 1,6 \\
\hline 6 & 1,00 & $-1,00$ & 1,00 & 1600 & 0,2000 & 0,8000 & 34,9 & 9,7 \\
\hline 7 & 1,00 & 1,00 & $-1,00$ & 1600 & 0,8000 & 0,2000 & 39,6 & 2,5 \\
\hline 8 & 1,00 & 1,00 & 1,00 & 1600 & 0,8000 & 0,8000 & 60,2 & 55,7 \\
\hline 9 & $-1,58$ & 0,00 & 0,00 & 54,98 & 0,5000 & 0,5000 & 62,2 & 61,0 \\
\hline 10 & 1,58 & 0,00 & 0,00 & 1945 & 0,5000 & 0,5000 & 63,9 & 65,5 \\
\hline 11 & 0,00 & $-1,58$ & 0,00 & 1000 & 0,0275 & 0,5000 & 5,8 & 85 \\
\hline 12 & 0,00 & 1,58 & 0,00 & 1000 & 0,9725 & 0,5000 & 59,9 & 55,2 \\
\hline 13 & 0,00 & 0,00 & $-1,58$ & 1000 & 0,5000 & 0,0275 & 63,9 & 65,5 \\
\hline 14 & 0,00 & 0,00 & 1,58 & 1000 & 0,5000 & 0,9725 & 56,6 & 46,5 \\
\hline 15 & 0,00 & 0,00 & 0,00 & 1000 & 0,5000 & 0,5000 & 61,6 & 59,5 \\
\hline 16 & 0,00 & 0,00 & 0,00 & 1000 & 0,5000 & 0,5000 & 63,2 & 63,6 \\
\hline 17 & 0,00 & 0,00 & 0,00 & 1000 & 0,5000 & 0,5000 & 61,1 & 58,2 \\
\hline 18 & 0,00 & 0,00 & 0,00 & 1000 & 0,5000 & 0,5000 & 63,8 & 65,2 \\
\hline 19 & 0,00 & 0,00 & 0,00 & 1000 & 0,5000 & 0,5000 & 61,4 & 58,9 \\
\hline 20 & 0,00 & 0,00 & 0,00 & 1000 & 0,5000 & 0,5000 & 62,1 & 60,8 \\
\hline 21 & 0,00 & 0,00 & 0,00 & 1000 & 0,5000 & 0,5000 & 62,4 & 61,5 \\
\hline
\end{tabular}




\section{1) LCOBEQ}

19 a 22 de outubro de 2014

Florianópolis/SC
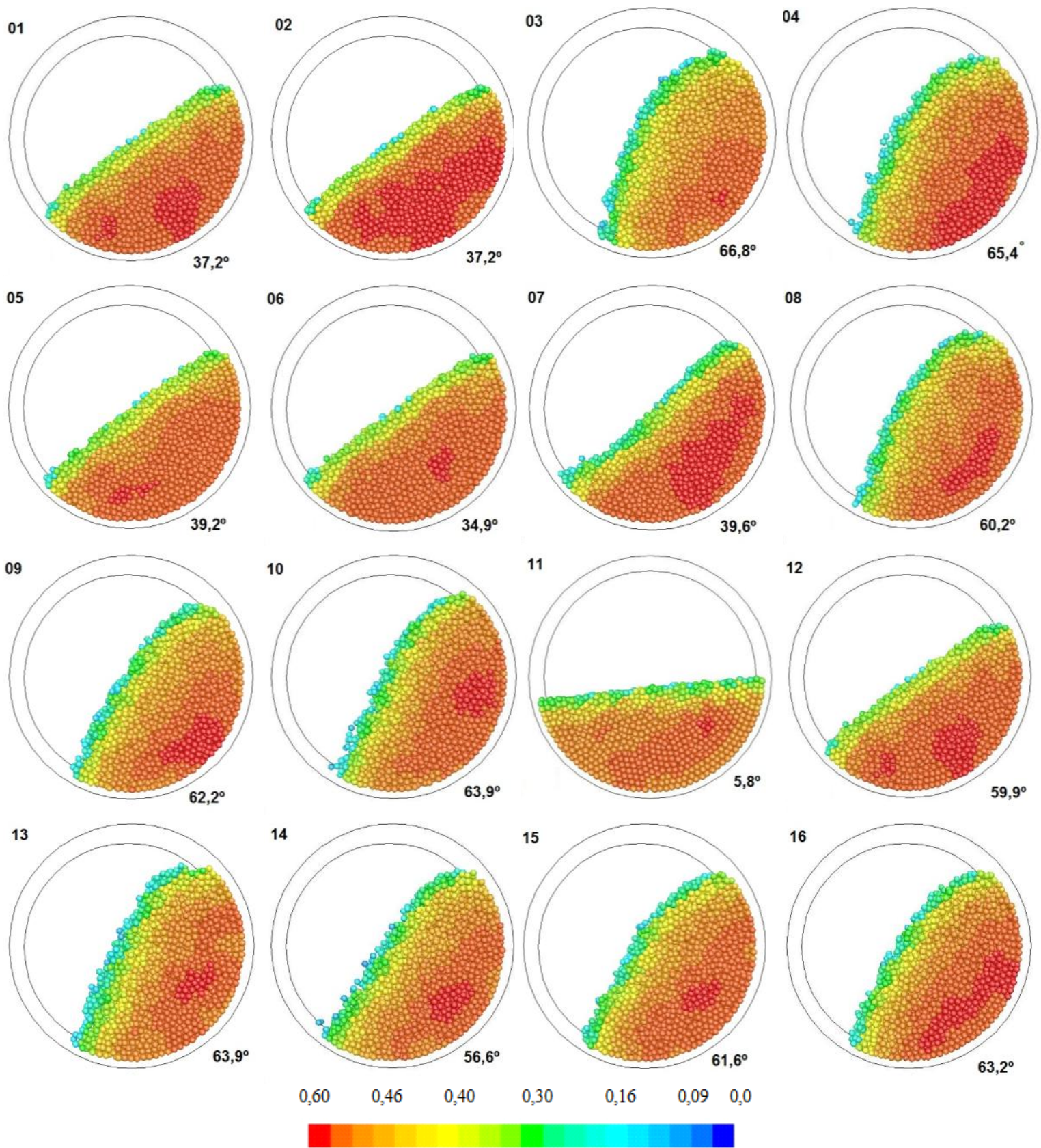

fração volumétrica de partículas

Figura 2 - Simulações DEM do tambor rotativo nas condições do PCC estudadas. 


\section{9 a 22 de outubro de 2014 \\ Florianópolis/SC}

\section{CONCLUSÕES}

Em geral, a técnica de simulação pelo DEM (método dos elementos discretos) mostrou resultados satisfatórios para a simulação do ângulo dinâmico de repouso em tambores rotativos e para a carga de sólidos nos suspensores em secadores rotatórios convencionais.

O conjunto de resultados de simulação pelo método dos elementos discretos (DEM) através do software FLUENT ANSYS® 14 quando comparados aos resultados experimentais de carga dos suspensores e ângulo dinâmico de repouso, mostraram melhores resultados para a seguinte combinação de parâmetros: constante de elasticidade $k$ igual a $400 \mathrm{~N} / \mathrm{m}$ e os parâmetros relacionados à fricção $\left(\mu_{f}\right)$ igual a 0,2 e à restituição $(\eta)$ iguais a 0,2 ou 0,8 ; ou seja, a simulação 01 e 02 do PCC ou a combinação da constante de elasticidade $k$ igual a $1600 \mathrm{~N} / \mathrm{m}$ e os parâmetros relacionados à restituição $(\eta)$ igual a 0,2 e à fricção $\left({ }^{\mu_{f}}\right)$ iguais a 0,2 ou 0,8 .

\section{AGRADECIMENTOS}

Agradecemos a Fundação de Amparo à Pesquisa do Estado de Goiás (FAPEG) pelos recursos concedidos no Edital de Participação em Eventos Científicos Nacionais (00032-2014) pelo auxilio financeiro a este trabalho.

\section{REFERÊNCIAS}

ANAND, A.; CURTIS, J. S.; WASSGREN,C.R.; HANCOCK,B.C.; KETTERHAGEN, W.R..Predicting discharge dynamics of wet cohesive particles from a rectangular hopper using the discrete element method (DEM). Chem. Eng. Sci. . v. 64 p. 5268-5275, 2009.

DI RENZO, A.; DI MAIO, F. P. Comparison of contact-force models for the simulation of collisions in DEM-based granular flow codes. Chem. Eng. Sci., v 59, p. 525 - 541, 2004.

KETTERHAGENA, W.R.; CURTIS, J.S.; WASSGREN, C.R.; HANCOCK, B.C.. Modeling granular segregation in flow from quasi-three-dimensional wedge shaped hoppers. Powder Technol. v. 179, p. 126-143, 2008.

LIU, P. Y.; YANG, N.; YU, A. B. DEM study of the transverse mixing of particles in rotating drums. Chem. Eng. Sci.. v 86, p. 99-107, 2012.

SANTOS, D. A. Contribuições ao estudo da Fluidodinâmica em leito de jorro - Estudos experimentais e de simulação via CFD. Universidade Federal de Uberlândia, Uberlândia - MG, 107p. Dissertação de mestrado, 2007.

ZHONG, W.; XIONG, Y.; YUAN, Z.; ZHANG, M.. DEM simulation of gas-solid flow behaviors in spouted-fluid bed. Chem. Eng. Sci.. v. 61, p. 1571-1584, 2006 